Following this psychophysical line of reasoning, it would be predicted that, using the Polidora and Thompson patterns, one would observe changes in discrimination acquisition if (1) the patterns initially contained fewer elements, or (2) greater increments of information were added, assuming, of course, that the basic pattern design is sensitive to these types of informational changes. At any rate, the present results are not to be interpreted as contradictory to any previous analyses, but should rather be viewed as supplemental to these findings and descriptive of a further dimension involving stimulus information and discrimination learning.

\section{REFERENCES}

LeVERE, T. E. Cue size and learning set performance with $S-R$ induced sampling biases eliminated. Journal of Comparative \& Physiological Psychology, 1968, 65, 362-365. LeVERE, T. E., \& BARTUS, R. T. APDA: A discontiguous $S-R$ automated primate discrimination apparatus. Behavioral Research Methodology \& Instrumentation, in press.
MACKINTOSH, N. J. Selective attention in animal discrimination learning. Psychological Bulletin, 1965, 64, 124-150.

MEYER, D. R., TREICHLER, F. R., \& MEYER, P. $M$. Discrete-trial training techniques and stimulus variables. In A. M. Schrier, H.F. Harlow, and F. Stollnitz (Eds.), Behavior of non-human primates Vol. 1. New York: Academic Press, 1965. Pp. 1-49.

POLIDORA, V. J., \& THOMPSON, W. J. Stimulus correlates of visual pattern discrimination by monkeys: Area and contour. Journal of Comparative \& Physiological Psychology, 1964 $58,264-269$.

SUTHERLAND, N. S. Visual discrimination in animals. British Medical Bulletin, 1964, 20, 54-59.

WILLIAMS, E. J. Experimental designs balanced for the estimation of residual effects of treatments. Australian Journal of Scientific Research, 1949, 2, 149-168. NOTES

1. This research was supported by Grant MH 14457-02 from the National Institute of Mental Health.

2. The authors wish to express thanks to C. A. LeVere for her help in the preparation of this report.

3. Information ratio is used here as a quantitative variable and describes the relationship between the amounts of information contained within the positive and negative stimulus displays.

\title{
Deprivation and the role of taste in food intake ${ }^{1}$
}

\author{
LOUIS A. HOFF, U.S. Army Natick \\ Laboratories, Natick, Mass. 01760, and \\ ROBERT L. GENTILE, Clark University, \\ Worcester, Mass. 01610
}

Rats were given single-stimulus feeding tests after varying degrees of food deprivation. Isocaloric diets containing different levels of quinine sulfate were used. Intake was measured after 30, 60, and $120 \mathrm{~min}$ of eating. At all deprivation levels and at all intervals, intake was negatively related to $\mathrm{QSO}_{4}$ concentration. While deprivation level increased intake of all diets, it did not influence relative intake among diets. These results do not support the view that the effects of taste on food intake change as a function of deprivation level.

It has been argued that the importance of taste is either attenuated or potentiated by deprivation. According to the hypothesis of attenuation, hunger is assumed to widen the range of acceptable food substances, increase the motivation to eat, and diminish the role of oral factors (Miller, 1962; Epstein, 1967). The hypothesis of potentiation suggests that the hungry animal is more, rather than less, discriminating. The importance of oral factors relative to caloric or metabolic signals is increased. This position predicts that, under conditions of deprivation, negative food substances diminish intake and positive substances increase it (Jacobs, 1966; Jacobs \& Sharma, in press).

Previous experiments relating to this topic have not been consistent in their use of criteria for defining attenuation or potentiation effects. Methods adopted have varied considerably and have not been amenable to direct comparison. Thus, one finds situations involving single foodcup tests in which a comparison between diets differing in taste quality is not made, direct choice-preference tests that may provide an entirely different situation to the organism, situations not directly involving food ingestion, and nonsystematic variations in deprivation measures (cf. Epstein, 1967; Jacobs \& Sharma, in press).

The present experiment attempts to assess the role of taste in intake behavior under conditions of single foodcup feeding using diets differing only in the quantity and quality of nonnutritive flavor substances used. Deprivation conditions were varied in a systematic fashion, comparisons between different test diets were carried out, and possible differences were evaluated.

\section{METHOD}

The Ss were 36 male Holtzman albino rats, ranging in age from 90-120 days. Ss were individually housed in $7 \times 7 \times 10$ in. stainless-steel cages, with wire-mesh floors and fronts. Water was available ad lib throughout all experiments. Food pellets (Wayne Lab Blox) were continuously available except during conditions of deprivation and testing. Conditions of temperature were controlled to $72 \operatorname{deg} F \pm 2 \operatorname{deg} F$.

The Ss were randomly divided into subgroups so that order of presentation of test diets and degree of deprivation could be counterbalanced.

The deprivation conditions used were 4 , 8,24 , and $48 \mathrm{~h}$ of food deprivation.

Test diets consisted of a wet mash base (Wayne Lab Meal), which was $50 \%$ tap water and, in addition, $0.1 \%$ quinine sulfate, N.F. $\left(\mathrm{QSO}_{4}\right)^{2}$ One diet $\left(\mathrm{H}_{2} \mathrm{O}\right)$ contained no additive. Thus, the diets were equated for calories but assumed different in taste quality. All diets were presented in spill-proof metal cups.

The actual procedure adopted involved dividing each subgroup into $\mathrm{H}_{2} \mathrm{O}$ and $\mathrm{QSO}_{4}$ sections and administering single foodcup feeding tests. Intake measures were taken after 30,60 , and $120 \mathrm{~min}$ of feeding, at which point the tests were terminated. One hour later, food pellets were provided ad lib to all Ss, and they were allowed a 2-day recovery period. Following this, the procedure was repeated with test diet or deprivation condition varied until Ss were measured under all experimental treatment conditions. Thus, order of presentation of test diets, order of deprivation degree, and daily variations in intake were assumed to be controlled for.

After this period of testing, the groups were given feeding tests with an additional diet consisting of the base diet with the addition of $0.2 \% \mathrm{QSO}_{4}$, using the same experimental procedure.

\section{RESULTS AND DISCUSSION}

Amounts of test diet eaten for each deprivation condition over the three time periods measured are depicted in Fig. 1. Deprivation level up to and including $24 \mathrm{~h}$ increased intake of all diets, while the 48-h groups did not significantly alter their intake of any diet relative to the 24-h intake. Such results are in accord with previous investigations (Miller, 1955; Dufort \& Wright, 1962; Dufort \& Lawler, 1968). At all deprivation levels and at all intervals, intake was negatively related to $\mathrm{QSO}_{4}$ concentration.

The shapes of the intake functions generated by the test diets over deprivation levels for all intervals are remarkably similar, pointing to the conclusion that the effects of taste are not altered by deprivation.

There are actually many criteria one might use in evaluating the effects of 


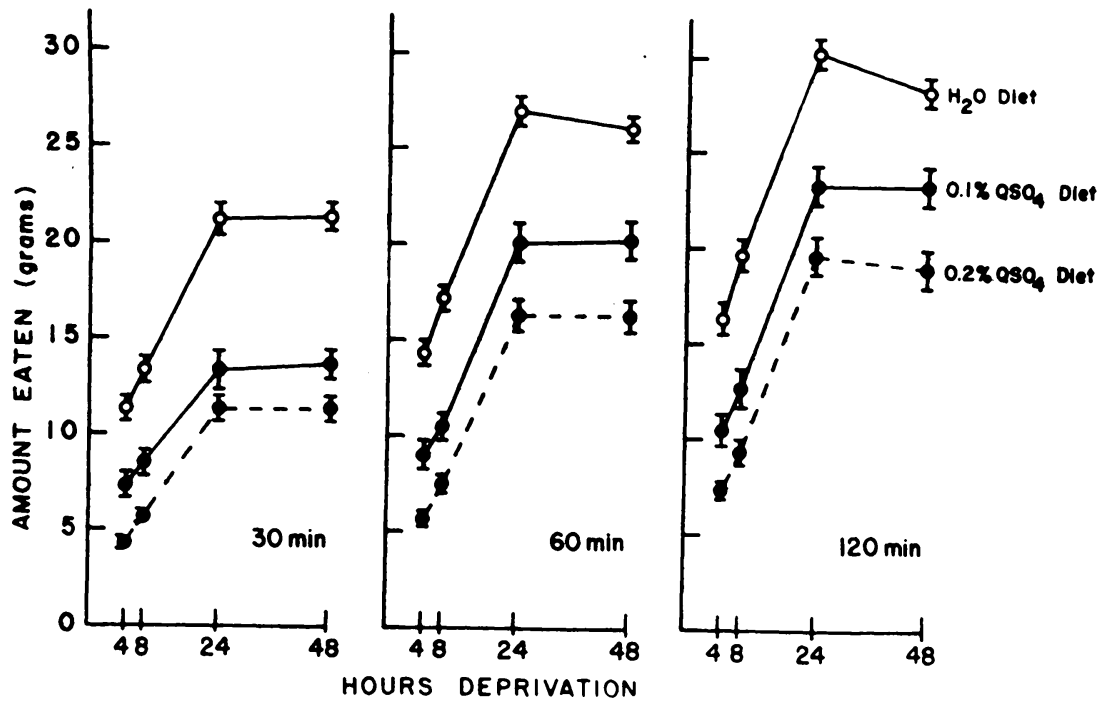

taste on intake. One might look at absolute intake, relative intake, or the difference scores between, diets. These types of measures have been commonly used in previous taste-effect experiments. Figure 2 depicts possible results of an intake experiment involving a palatable $\left(\mathrm{H}_{2} \mathrm{O}\right)$ and an unpalatable $\left(\mathrm{QSO}_{4}\right)$ diet and deprivation levels, as used in the present experiment. The data points for $\mathrm{H}_{2} \mathrm{O}$ diet represent a function generated by intake at all intervals, as observed in the present experiment. The $\mathrm{QSO}_{4}$ diet intake levels represent hypothetical functions that could be interpreted as exhibiting a constant taste effect. The function $Q=K$ represents a constant taste effect on absolute intake, a measure of absolute acceptability. As deprivation increases, the intake of an unpalatable diet does not change. The function $H / Q=K$ defines taste effect constancy in terms of a comparison of palatable and unpalatable diet intakes, a measure of relative acceptability. As deprivation increases, the intake ratio of palatable diet to unpalatable diet is constant. The function $H-Q=K$ defines taste-effect constancy by comparing the diets in terms of difference scores, a measure of relative rejection; it states that the difference between amounts of palatable and unpalatable diets eaten over deprivation conditions is constant.

Since the above functions define all of the possible results that could be interpreted as exhibiting a constant taste effect in terms of previously accepted criteria, results that vary from the above could provide evidence for a change in the importance of taste over deprivation conditions.

Increasing acceptance of an unpalatable diet has often been taken to mean an increase in absolute intake, implying that constancy would be defined by the line $Q=K$, i.e., constant intake of the unpalatable diet over deprivation conditions. However, since deprivation is known generally to increase food intake, it might be in error to assume that an increase in unpalatable-diet intake is an adequate criterion for evidence of attenuation of taste effect per se. It seems more reasonable, therefore, to attempt

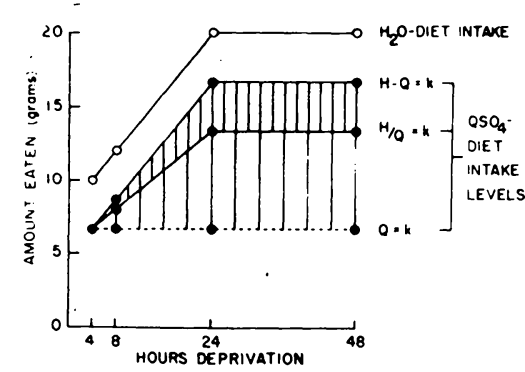

Fig. 2. Hypothetical intake functions of palatable $\left(\mathrm{H}_{2} \mathrm{O}\right)$ and unpalatable $\left(\mathrm{QSO}_{4}\right)$ diets as a function of hours of deprivation.
Fig. 1. Amount of test diet eaten as a function of hours of deprivation over the three intake periods.

comparisons of test diets in order to test the hypothesis.

All of the data obtained in the present experiment lie between the points generated by the function $H / Q=K$, a constant ratio of diet intake under varying degrees of deprivation, and $H-Q=K, a$ constant difference.

It seems, therefore, that a case can be made for constant effects across diets in terms of relative acceptability or relative rejection. Using these criteria, it appears that, while palatability and deprivation influence the amount eaten, relative intake functions remain the same. Thus, the importance of taste relative to other aspects of a food stimulus appears unchanged by deprivation, at least in the single foodcup situation.

\section{REFERENCES}

DUFORT, R. H., \& WRIGHT, J. H. Food intake as a function of duration deprivation. Journal of Psychology, 1962, 53, 465-468.

DUFORT, R. H., \& LAWLER, J. E. Food intake with and without water after different durations of food deprivation. Psychological Reports, 1968, 22, 905-908.

EPSTEIN, A. E. Oropharyngeal factors in feeding and drinking. In C. F. Code and W. Heidel (Eds.), Handbook of physiology, Section 6 : Alimentary canal. Vol. 1. Washington: American Physiological Society, 1967. Pp. 197-218.

JACOBS, H. L. Sensory and metabolic regulation of food intake: Thoughts on a dual system regulated by energy balance. Proceedings of the Seventh International Congress of Nutrition. Vol. 2. Hamburg, 1966. Pp. 3-15.

JACOBS, H. L., \& SHARMA, K. N. Taste versus calories: Sensory and metabolic signals in the control of food intake. Annals of the New York Academy of Science, in press.

MILLER, N. E. Shortcomings of food consumption as a measure of hunger: Results from other behavioral techniques. Annals of the New York Academy of Science, 1955, 63, 141-143.

MILLER, N. E. Discussion. In Proceedings of the International Union of Physiological Sciences. Vol. 1. XXII International Congress. Amsterdam: Excerpta Medica Foundation, 1962. Pp. 705-707.

\section{NOTES}

1. This research was carried out at the Pioneering Research Laboratory, Behavioral Sciences Division, U.S. A rmy Natick Laboratories, Natick, Mass. 01760.

2. Quinine sulfate from Mallinckrodt Chemical Works, N.Y. (Mallinckrodt No. 1891). 